Monatsschr Kinderheilkd 2021 • 169:1075-1082 https://doi.org/10.1007/s00112-021-01159-z Angenommen: 3. März 2021

Online publiziert: 3. Mai 2021

(c) Der/die Autor(en) 2021

\section{Redaktion}

A. Borkhardt, Düsseldorf

S. Wirth, Wuppertal

S. Dillenhöfer ${ }^{1} \cdot$ B. Hinrichs ${ }^{2} \cdot$ A. Kohl ${ }^{3} \cdot$ M. Kuhnigk ${ }^{4} \cdot$ R. Maas ${ }^{5} \cdot$ D. Pfeiffer-Kascha ${ }^{6}$. T. Rutt ${ }^{7} \cdot$ A. Schlegtendal ${ }^{1} \cdot$ J. Seidenberg ${ }^{8} \cdot$ T. Spindler $^{9} \cdot$ C. Suerbaum ${ }^{10}$.

B. Wilmsmeyer ${ }^{11} \cdot$ S. Zeidler ${ }^{12} \cdot$ C. Koerner-Rettberg ${ }^{1,13}$

' Universitätsklinik für Kinder- und Jugendmedizin, Katholisches Klinikum Bochum, Klinik der RuhrUniversität Bochum, Bochum, Deutschland

${ }_{2}^{2}$ Praxis Lindenstraße, Buchholz, Deutschland

${ }^{3}$ Kinderlungenpraxis, Kiel, Deutschland

${ }^{4}$ Klinik für Kinder- und Jugendmedizin, Marienhospital Bottrop, Bottrop, Deutschland

${ }^{5}$ Kinder- und Jugendpsychiatrie, Psychotherapie und Psychosomatik, Klinikum Frankfurt (Oder), Frankfurt (Oder), Deutschland

${ }^{6}$ Physiotherapie-Praxis Röske, Wuppertal, Deutschland

${ }^{7}$ Praxis Kinder- und Jugendmedizin, Hamburg, Deutschland

${ }^{8}$ Medizinische Fakultät, Bereich Pädiatrie, Universität Oldenburg und Göttingen, Carl von Ossietzky Universität Oldenburg, Oldenburg, Deutschland

${ }^{9}$ Hochgebirgsklinik Davos, Davos Wolfgang, Schweiz

${ }^{10}$ Schwerpunktpraxis Pneumologie, Düsseldorf, Deutschland

"Kinderpneumologische Praxis Itzehoe, Itzehoe, Deutschland

${ }^{12}$ Asklepios Klinik Sankt Augustin, Sankt Augustin, Deutschland

${ }^{13}$ Klinik für Kinder- und Jugendmedizin, Marien-Hospital Wesel, Wesel, Deutschland

\title{
Die induzierbare laryngeale Obstruktion (ILO) - Ursachen, klinische Präsentation, Diagnostik und Therapie
}

\section{Positionspaper der Arbeitsgruppe „Dysfunktionelle Respiratorische Symptome" der Gesellschaft für Pädiatrische Pneumologie (GPP)}

\author{
gut behandelbaren Entitäten noch \\ unzureichend.
}

\section{Ziel des Positionspapiers} ner hohen Prävalenz von 5-10\% spielen funktionelle Atemstörungen mit respiratorischen Symptomen eine zahlenmäßig große und bisher unterschätzte Rolle: Sie werden häufig lange verkannt und als Asthma fehlbehandelt. Dagegen sind andere somatische Ursachen (u.a. Atemwegsfehlbildungen) deutlich seltener. Trotz zunehmender Evidenz zur "inducible laryngeal obstruction" (ILO) sind die Bekanntheit und das Bewusstsein für diese
Eine ILO ist ein häufiges Störungsbild innerhalb des Spektrums funktioneller Atemstörungen [1]. Die Autoren haben zum Ziel, auf der Basis des aktuellen Wissensstandes konkrete Handlungsempfehlungen für Diagnostik und Therapie paroxysmaler induzierbarer laryngealer Obstruktionen (ILO) auszusprechen, dabei die vorhandenen diagnostischen Ressourcen zu berücksichtigen und bestehende Erkenntnislücken zu benennen. Es findet die aktuelle von der European
Respiratory Society (ERS) und European Laryngological Society (ELS) 2017 [2] empfohlene Nomenklatur Berücksichtigung, deren Benutzung empfohlen wird. Die vorliegende Arbeit soll für die häufigen funktionellen Atemstörungen sensibilisieren, um inadäquate AsthmaTherapien und lange Leidenswege bis zur ILO-Diagnosestellung zu reduzieren. Es soll Betroffenen eine wirksame Therapie eröffnet sowie die Lebensqualität verbessert werden.

\section{Systematik und Terminologie}

Die Symptomatik ,inspiratorische Atemnot mit charakteristischem Atemge- 


\section{Konsensuspapiere}
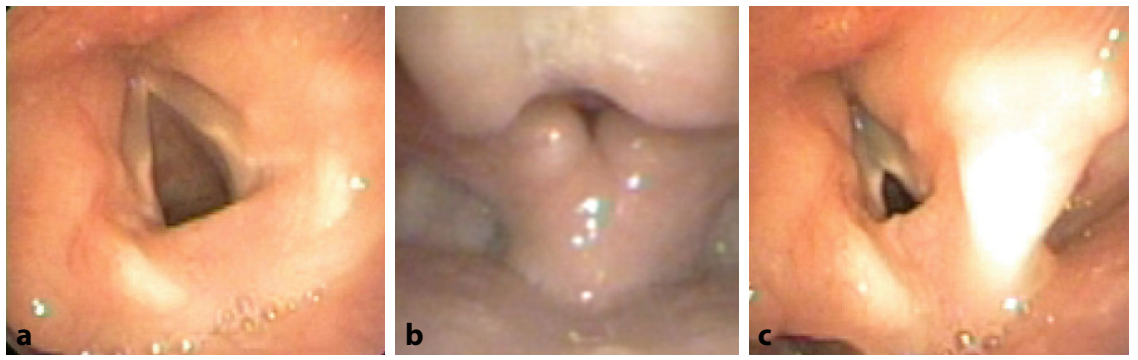

Abb. 1 A Laryngoskopische Sicht auf Glottis und supraglottische Strukturen; a Normalbefund, b Bild einer supraglottischen „exercise-induced laryngeal obstruction" (EILO) in Inspiration, c Bild einer "Vocal cord dysfunction" (VCD) während der Inspiration. (৫ Prof. J. Seidenberg, alle Rechte vorbehalten. Abdruck mit freundl. Genehmigung)

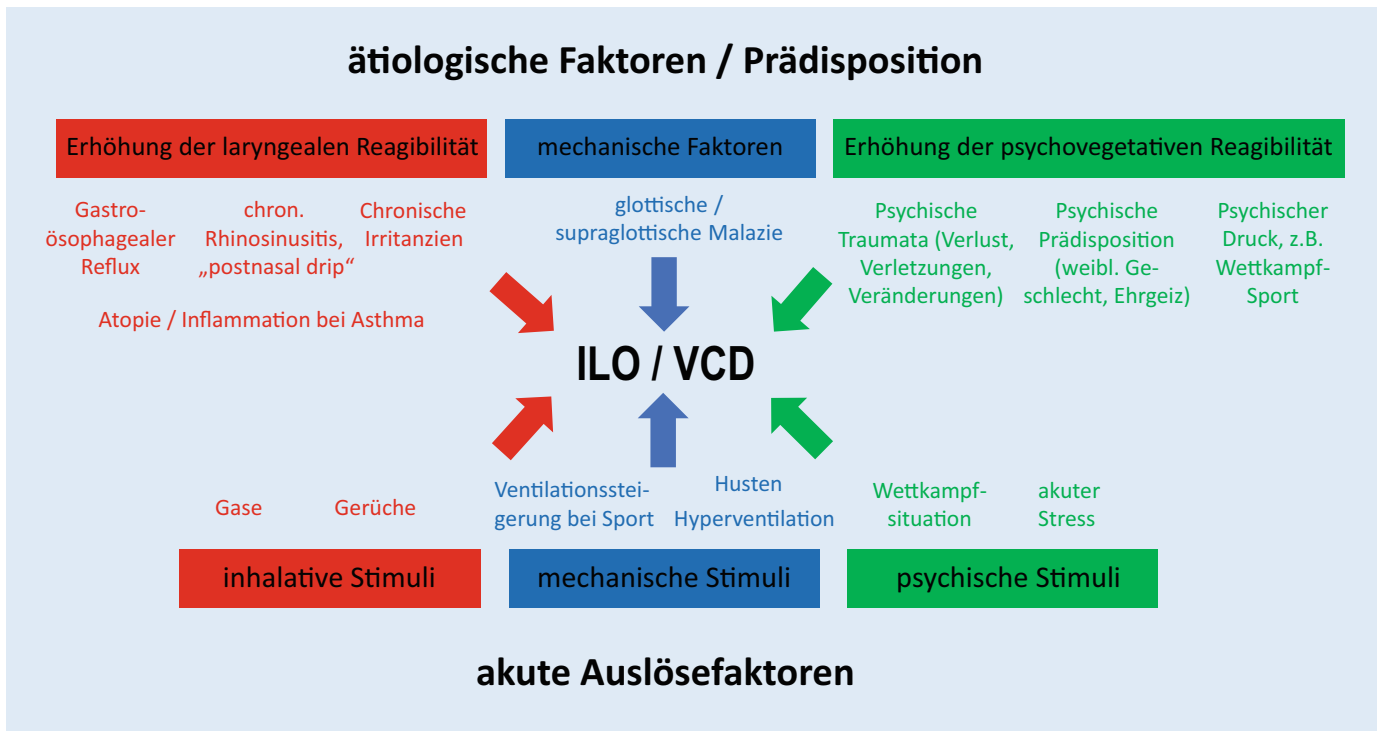

Abb. $2<1$ ILO-Pathogenese-Konzept

räusch“ - verursacht durch eine transiente reversible Obstruktion des Larynx wurde in der Vergangenheit mit den unterschiedlichsten Termini benannt, wie z. B. laryngeale Neuropathie, Syndrom des irritablen Larynx, inspiratorischer Glottiskrampf, „paradoxical vocal fold motion“, „paradoxical vocal cord dysfunction“, „upper airway obstruction“, „exercise-induced laryngomalacia“, „laryngeal dysfunction“ etc. Der Terminus „Vocal cord dysfunction“ (VCD) ist im Laufe der Jahrzehnte in der Literatur zur dominierenden Bezeichnung geworden. Streng genommen beschreibt der Begriff VCD lediglich eine induzierbare laryngeale Obstruktion auf Glottisebene [3]. Mittlerweile ist auch die Existenz supraglottischer obstruktiver Funktionsstörungen gut belegt. Aufgrund dieser Ungenauigkeit hat die ERS zusammen mit der ELS und dem American College of Chest Physicians als internationale
Task Force 2013 den Oberbegriff, ,inducible laryngeal obstruction“ = induzierbare laryngeale Obstruktion - ILO gewählt [4].

Symptome einer ILO können sowohl anstrengungsinduziert als auch spontan in Ruhe oder durch andere Auslöser (Gerüche, psychische Trigger) ausgelöst werden. Für die anstrengungsinduzierte Form wurde der Oberbegriff „exerciseinduced laryngeal obstruction“ (EILO) geprägt $[2,4]$. Diese kann sowohl auf glottischer Ebene als anstrengungsinduzierte paradoxe Stimmbandbewegung („,exercise-induced VCD“ (EI-VCD)) als auch auf supraglottischer Ebene als anstrengungsinduzierte Laryngomalazie („,exercise-induced laryngomalacia“ (EILM)) mit Anteversion und Kippung der Aryknorpel unter Belastung stattfinden (- Abb. 1). Die Benutzung des Terminus VCD kann daher, streng genommen, nur nach laryngoskopisch festgestellter para- doxer Stimmlippenbewegung verwendet werden, ansonsten sollte der Begriff ILO - bzw. bei Auslösung durch körperliche Anstrengung EILO - gewählt werden [4].

\section{Prävalenz und Risikogruppen}

Es gibt keine kontrollierten Studien zur Häufigkeit der ILO. Angaben zur Prävalenz reichen von $5,7 \%$ unter unselektierten Adoleszenten [5] bzw. 15\% EILO-Prävalenz bei Armee-Rekruten [6] bis maximal $27 \%$ bei Kindern und Jugendlichen mit unkontrolliertem Asthma [7, 8]. Auch wenn nach Erfahrung der Autoren Jugendliche und junge Erwachsene besonders häufig betroffen sind, kann eine EILO durchaus auch bereits vor dem Pubertätsalter auftreten. $\mathrm{Ab}$ der Pubertät überwiegt das weibliche Geschlecht (Verhältnis m:w 1:2 bis 1:3 [9-11]). Unter einer EILO leiden häufig 
auch Leistungssportler [12, 13]. Bei der nichtanstrengungsinduzierten VCD sind häufiger junge Frauen mit psychiatrischen Krankheitsbildern (z. B. Angststörungen, Konversionssyndromen) nach Traumata und/oder Gewalterfahrung betroffen [14-17]. Bei Jugendlichen mit ILO konnte ein Zusammenhang mit sozialem Stress und Angst gezeigt werden [18]. Daneben gibt es Hinweise auf somatische prädestinierende bzw. auslösende Faktoren wie die chronische Rhinosinusitis [19] und den gastroösophagealen bzw. laryngopharyngealen Reflux [9, 18, 20-23].

\section{Ätiologie und Pathogenese}

Die Ätiologie der ILO ist komplex, multifaktoriell (-Abb. 2; [24]) und noch nicht klar verstanden. Das aktuelle Pathogenese-Konzept geht von einer Prädisposition durch

a) laryngeale Hyperreagibilität,

b) erhöhte psychovegetative Vulnerabilität und/oder

c) mechanische Faktoren aus.

In der Literatur wird ursächlich ein pathologisch gesteigerter Larynxreflex, der als Schutzmechanismus eine durchaus physiologische Rolle hat, beschrieben, ebenso wie eine exogen induzierte laryngeale Hyperreagibilität. Die lokale laryngeale Reagibilität kann z. B. durch einen laryngopharyngealen Reflux, eine chronische Rhinosinusitis, chronische Exposition gegenüber Irritanzien (Reinigungs-/Lösungsmittel, Rauch) [25] oder im Rahmen einer allgemeinen Schleimhaut-Hyperreagibilität z. B. bei Asthma, erhöht sein [26]. Es gibt Hinweise dafür, dass bereits ein leicht-saurer Reflux mit laryngopharyngealer Komponente (LPR) die Larynx-Schleimhaut schädigen $[9,20]$, auch ohne eine ösophageale Symptomatik zu verursachen. Der Zusammenhang zwischen Reflux und Folgen an der Larynx-Schleimhaut ist beschrieben [27, 28], und in einigen VCD-Fallserien ist die Beobachtung eines Benefits von einer medikamentösen Refluxtherapie festgehalten [9, 29, 30]. Bei der chronischen Rhinosinusitis wird eine Larynx-Reizung durch einen „postnasal drip“ des Entzündungssekrets als

Monatsschr Kinderheilkd 2021 · 169:1075-1082 https://doi.org/10.1007/s00112-021-01159-z (c) Der/die Autor(en) 2021

S. Dillenhöfer - B. Hinrichs - A. Kohl · M. Kuhnigk - R. Maas · D. Pfeiffer-Kascha · T. Rutt · A. Schlegtendal $\cdot$ J. Seidenberg $\cdot$ T. Spindler $\cdot$ C. Suerbaum $\cdot$ B. Wilmsmeyer $\cdot$ S. Zeidler . C. Koerner-Rettberg

Die induzierbare laryngeale Obstruktion (ILO) - Ursachen, klinische Präsentation, Diagnostik und Therapie. Positionspaper der Arbeitsgruppe „Dysfunktionelle Respiratorische Symptome" der Gesellschaft für Pädiatrische Pneumologie (GPP)

\section{Zusammenfassung}

Wiederholt episodenhaft auftretende Atemnot ist im Kindes- und Jugendalter ein häufiges Symptom. Neben anstrengungsinduzierter Bronchialobstruktion im Rahmen eines Asthma bronchiale ist eine funktionelle Genese eine sehr häufige Differenzialdiagnose. Dennoch wird diese Diagnose häufig nicht oder mit langer Latenz gestellt. Unter dem Oberbegriff „ILO“ („inducible laryngeal obstruction“) werden sowohl funktionelle supraglottische Obstruktionen durch Kollaps der Knorpelstrukturen als auch Dysfunktionen auf Glottisebene wie "vocal cord dysfunction" (VCD) subsumiert. Körperliche Anstrengung ist ein häufiger Auslöser; es werden jedoch auch Beschwerdebilder ohne Anstrengungsbezug beobachtet. Es wird der Erkenntnisstand zur Pathophysiologie referiert und die klinische Präsentation beschrieben. Ein wesentlicher
Fokus des Artikels liegt im Folgenden auf der Darstellung eines sinnvollen und Ressourcenorientierten diagnostischen Vorgehens. Der CLE-Test ("continuous laryngoscopy exercise test ${ }^{\prime \prime}$ ) als Provokationsmethode unter WachVideolaryngoskopie ist der Goldstandard, jedoch wird diese Diagnostik im deutschsprachigen Raum nicht flächendeckend vorgehalten. Dieses Positionspapier stellt daher die diagnostische Wertigkeit verschiedener anderer Diagnostik-Algorithmen und anamnestischer Informationen heraus. Ein weiterer Schwerpunkt des Papiers besteht in der detaillierten Vorstellung geeigneter atemphysiotherapeutischer Interventionen.

\section{Schlüsselwörter}

Dyspnoe · Atem-Physiotherapie · „Vocal cord dysfunction" · Dysfunktionelles Atemmuster . Laufband-Provokation

\section{Inducible laryngeal obstruction (ILO)-Causes, clinical presentation, diagnostics and treatment. Position paper of the working group on dysfunctional respiratory symptoms of the Society for Pediatric Pneumology (GPP)}

\section{Abstract}

Episodically occurring dyspnea has a high prevalence in childhood and adolescence. Besides exercise-induced bronchial obstruction in a child with asthma, functional respiratory problems are also frequently encountered; however, in cases of functional disorders the correct diagnosis is often not made or made after a long latent period. The term inducible laryngeal obstruction (ILO) comprises functional supraglottic obstruction due to cartilage collapse as well as glottic disorders, such as vocal cord dysfunction (VCD). Physical exertion is a common trigger but there are also other entities which occur without relation to exercise. One main goal of the article is to delineate a reasonable diagnostic pathway taking the limited access to a continuous laryngoscopy exercise (CLE) test in Germany into account: The CLE test is presently done by a treadmill provocation test while visualizing the larynx with a direct laryngoscopy; however, this procedure is not widely available in Germany. Hence the authors of this paper discuss the diagnostic power of other procedures and suggest a reasonable diagnostic algorithm. A second focus of the paper is on a detailed presentation and discussion of suitable physiotherapeutic interventions.

\section{Keywords}

Dyspnea $\cdot$ Physiotherapeutic intervention . Vocal cord dysfunction - Dysfunctional breathing pattern - Treadmill provocation 
Tab. 1 Charakteristika von „exercise-induced laryngeal obstruction" (EILO) versus „exercise-induced bronchoconstriction" (EIB)

EILO

Atemgeräusch Inspiratorisch (meistens)

Lokalisation Larynx

Dauer Eine bis wenige Minuten, selten länger

Beschwerde-Beginn 1,5-2 min nach Beginn körperlicher Belastung

Beschwerde-Ende Rasch nach Beendigung körperlicher Belastung

$\beta_{2}$-Mimetika

Unwirksam

Spirometrie
F/V-Kurve normal oder mit inspiratorischen Oszillationen, evtl. abgeflacht
EIB

Exspiratorisch

Bronchien

Variabel

5-10 min nach Beginn körperlicher Belastung

Variabel

Wirksam

F/V-Kurve mit konkav verformter Exspirationskurve
Wegbereiter für ILO-Symptome postuliert [19]. Dieses wird durch einen hohen Anteil von Patienten mit chronischer Rhinosinusitis mit laryngealer Hyperreagibilität auf Methacholin-Inhalation untermauert.

Daneben scheinen psychosoziale Begleitumstände oder Vulnerabilitätsfaktoren eine wichtige Rolle zu spielen [14-18, 23]. Eine Erhöhung der psychovegetativen Vulnerabilität resultiert aus emotionalen Traumata, psychischer Prädisposition und/oder psychischem Druck durch z. B. Wettkampf-Sport [12, 13, 18].

In der Literatur wurde bei VCD-Patienten eine höhere Prävalenz von Asthma bronchiale als in der Allgemeinbevölkerung beschrieben [31, 32]. Da dies unabhängig vom Asthmaschweregrad zu sein scheint, lässt dies vermuten, dass die Schleimhaut-Entzündung pathogenetisch weniger bedeutend ist als das $\mathrm{Zu}$ sammenwirken psychovegetativer Faktoren. Denkbar ist ein „Erinnerungseffekt" für das Symptom Atemnot. Eine unzureichende Asthmakontrolle trotz adäquater Therapie sollte insbesondere bei Vorhandensein suggestiver Symptome den Verdacht auf eine zusätzliche ILO-Komponente lenken. Daher ist es wichtig, typische Symptome (z.B. Stridor) aktiv nachzufragen. Um Missverständnisse zu vermeiden, sollte dem Patienten anhand eines Videos oder durch den behandelnden Arzt selbst ein inspiratorischer Stridor demonstriert werden. Sehr hilfreich sind auch kurze Filmsequenzen, die Betroffene im Rahmen der Episoden von sich aufnehmen lassen.

Bei den mechanischen Faktoren wird eine strukturelle laryngeale Malazie diskutiert, entsprechend der Laryngomalazie im Säuglingsalter. Diese ist jedoch als Risikofaktor für eine spätere EILO bisher nur in einer Publikation mit klei- ner Patientenzahl beschrieben [33]. Außerdem mag der bei der EILO beobachtete Kollaps laryngealer Strukturen auch durch ein pathologisches Atemmuster bedingt sein. Hierfür spricht die gute atemphysiotherapeutische Beeinflussbarkeit der Symptome bis hin zur vollständigen Beschwerdefreiheit bei EILOPatienten ([34, 35] und Erfahrungen der Autoren).

Auf dem Boden dieser beschriebenen prädisponierenden Faktoren können akute Auslöser zu den ILO-Symptomen führen. Hierzu gehören Hyperventilation, Husten, Aspiration, Inhalation von chemischen Irritanzien (Gerüche, Rauch etc.), körperliche Anstrengung und psychische Trigger $([5,14,25]$ und Review bei [23]).

\section{Klinisches Bild}

Typisch für eine ILO ist ein plötzlicher Beginn mit rasch zunehmender Atemnot, welche bei der EILO durch körperliche Anstrengung ausgelöst wird. Die Atemnot wird vorwiegend als inspiratorisch beschrieben. Betroffene berichten oft, dass „sich der Hals zuschnürt“ oder „keine Luft reingeht“. Juguläre Einziehungen mit Einsatz der Atemhilfsmuskulatur sind sichtbar. Die Inspiration kann verlängert sein und wird meist begleitet von einem hochfrequenten, stridorösen Atemgeräusch. Selten ist der Stridor biphasisch, also auch bei der Exspiration hörbar. Manchmal kommt es nur zu einer verstärkten „keuchenden“ Einatmung ohne klassischen Stridor. Der Geräuschcharakter scheint dabei von der Obstruktionsebene (supraglottisch versus glottisch) abhängig $\mathrm{zu}$ sein. Im Gegensatz zur „exercise-induced bronchoconstriction" (EIB) beim Asthma bronchiale tritt die Atemnot bei der EILO bereits während und nicht nach der körperlichen Belastung auf (•Tab. 1). Während EILO-Symptome typischerweise eine kurze Dauer von wenigen Minuten haben und bei Beendigung der Belastung rasch sistieren, persistieren Symptome durch eine bronchiale Obstruktion deutlich länger. Allerdings kann eine VCD-Episode ohne Anstrengungsbezug gelegentlich auch Stunden bis Tage andauern (eigene Beobachtungen). Dabei können die Patienten typischerweise trotz offensichtlicher signifikanter Atemnot noch gut sprechen. Zusätzlich können zeitgleich Symptome weiterer funktioneller Atembeschwerden wie eine Hyperventilation oder eine Seufzerdyspnoe, aber auch nichtrespiratorische Beschwerden wie Kopfschmerzen, Übelkeit oder Panikattacken auftreten. Gelegentlich wird auch ein lokalisierter Schmerz oder Druck in der Brust oder im Halsbereich (Globusgefühl) angegeben.

ILO-Attacken können zu sekundären, zum Teil schweren, psychischen Beeinträchtigungen wie Angst- oder Panikstörungen bis hin zu einer reaktiven Depression führen, insbesondere bei der VCD [36].

\section{Diagnostisches Vorgehen}

Ein Verdacht auf eine ILO begründet sich typischerweise durch eines oder mehrere der folgenden Merkmale, sodass die Anamnese hierauf ausgerichtet sein sollte [37, 38]:

- nicht hinreichend auf Asthmamedikation ansprechende AtemnotEpisoden bei Asthma bronchiale,

- Atemnot durch erschwerte Inspiration, 
- inspiratorisch hörbares lautes Atemgeräusch bis hin zum klassischen Stridor,

- plötzlicher Beginn der Symptome aus dem Nichts heraus (bei VCD),

- Atemnot isoliert bei Belastung (ohne Auftreten bei anderen typischen Asthma-Beschwerdeauslösern wie Infekten oder Inhalationsallergenexposition),

- bei EILO: kurze Dauer (wenige Minuten) mit raschem Sistieren bei Belastungsabbruch,

- Fehlen von Symptomen im Schlaf.

Neben der Ermutigung zu einem freien Bericht des Patienten mit Beschreibung seiner Symptome in eigenen Worten sollten bestimmte gezielte, informative Fragen gestellt werden:

- Ist die Atemnot in- oder exspiratorisch?

- Empfindet der Patient mit vorbestehendem Asthma verschiedene Arten von Atemnot?

- Kann der Patient die Einengung der Atemwege auf eine Körperstelle (z. B. Hals) lokalisieren?

- Tritt die Atemnot plötzlich von einem Moment zum nächsten auf?

- Im Falle eines auftretenden Atemgeräusches: Tritt dieses bei der Einoder Ausatmung auf?

- Gibt es bestimmte Auslöser wie Husten, Gerüche, Stress, Anstrengung etc.?

- Tritt die Atemnot isoliert bei körperlicher Belastung auf?

- Tritt Atemnot während oder erst nach der Belastung auf?

- Wie schnell sistiert sie nach Belastungsabbruch?

- Wird das Asthma-Notfallspray (Salbutamol) als wirksam empfunden? Wenn ja: wie schnell?

- Was hilft dem Patienten, um die Atemnot $\mathrm{zu}$ vermeiden oder $\mathrm{zu}$ beenden?

- Gibt es begleitende Beschwerden wie z.B. Husten, Schwindel oder Kribbeln/Verkrampfung in den Fingern?

- Besteht Heiserkeit oder eine Stimmveränderung während der Episode?
Da die Patienten bei der Vorstellung häufig asymptomatisch sind und die Anamneseerhebung die Diagnose nicht immer klar festlegen lässt, sollten die Betroffenen aufgefordert werden, bei Beschwerden Filmaufnahmen zu erstellen (z. B. mit dem Smartphone) und diese bei dem nächsten Vorstellungstermin vorzuzeigen.

Wenn das Symptom Atemnot isoliert bei Belastung (nie im Zusammenhang mit anderen Asthma-typischen Auslösern wie Infekten oder Allergenexposition) auftritt, sollte die Einordnung als Asthma-bedingte Atemnot a priori kritisch zugunsten einer EILO hinterfragt werden.

In der Hand eines erfahrenen Pneumologen kann die Diagnose einer ILO bereits durch diese anamnestischen Hinweise hochgradig wahrscheinlich gemacht werden, und es mag dann in einigen Fällen eine Laufband-Provokation zur Diagnose-Sicherung entbehrlich sein. In der Realität wird jedoch weiterhin die Diagnose einer ILO deutlich zu selten oder mit großer zeitlicher Latenz gestellt. Eine einfache LaufbandProvokation, in der EILO-Symptome evoziert werden, bei gleichzeitigem Ausschluss einer Bronchialobstruktion, ist daher - auch ohne gleichzeitige Laryngoskopie per CLE-Test (s. unten) - ein geeignetes Mittel zur Diagnosestellung. Zudem kann die Erläuterung der Untersuchungsergebnisse der LaufbandProvokation die Bereitschaft des Patienten zur Annahme der Diagnose gut unterstützen.

Zur Abgrenzung gegenüber unzureichend kontrolliertem Asthma bronchiale und zum Ausschluss fixierter Atemwegsstenosen sollte auch eine Ruhe-Lungenfunktion mit forcierter In- und Exspiration, ggf. mit Bronchospasmolyse, erfolgen. Bei der Interpretation der Lungenfunktion ist zu beachten, dass es zwar als VCD-typisch beschriebene Phänomene gibt (wie Unfähigkeit des Patienten, repetitiv gleichwertige Lungenfunktionskurven zu produzieren oder eine abgeflachte Inspirationskurve [23]), eine unauffällige Ruhe-Lungenfunktion jedoch weder eine ILO noch ein Asthma bronchiale ausschließt. Bei einer auffälligen Lungenfunktion müssen weitere Ursachen wie z. B. eine Kompression der Atemwege durch aberrant verlaufende Gefäße oder Raumforderungen wie z.B. eine Struma differenzialdiagnostisch ausgeschlossen werden. Eine „typische“ Lungenfunktion für die Diagnose „ILO“ gibt es nicht.

Ein Laufband-Provokationstest mit einem individuell gewählten Belastungsniveau, geeignet zum Auslösen von Symptomen, dient zur Differenzierung zwischen einer Asthma-assoziierten Bronchokonstriktion (EIB) und einer EILO. Die Beobachtung muss durch eine hierin fachkundige Person erfolgen. Beurteilt werden die Zeit bis zum Auftreten von Atemnot, Vorhandensein und Charakteristik von Atemgeräuschen, Körperhaltung und Atemmuster (thorakal dominierte Atemexkursion, Schultern-Hochziehen bei Inspiration). Eine Objektivierung von Beobachtungen des Atemmusters ist wünschenswert, jedoch fehlen hierzu bisher im Alltag nutzbare technische Lösungen. Eine Methacholin-Provokation kann nicht nur eine EIB, sondern auch eine ILO auslösen [2], ist jedoch bezüglich ihrer Fähigkeit, zwischen den zwei Entitäten zu differenzieren, nicht ausreichend untersucht und daher nicht empfehlenswert.

Die Beobachtung des Patienten muss in jedem Fall nach Belastungsende fortgesetzt werden, um weitere Symptome wie eine Hyperventilation und die typischerweise erst nach Ende der Belastung auftretenden Symptome und Lungenfunktionsveränderungen einer EIB erfassen $\mathrm{zu}$ können. Während eine EIB oft erst nach 5-10 min beginnt (und daher auch Lungenfunktionsmessungen zu diesem Zeitpunkt erforderlich sind), kommt es zu EILO-Symptomen meist schon während, typischerweise kurz (1,5-2 min) nach Beginn der Belastung, um dann rasch nach Beendigung der Belastung wieder abzuklingen. Bei einer EILO findet sich im Gegensatz zum Asthma keine Bronchialobstruktion in der Lungenfunktion.

Zusätzlich sollte auf das Auftreten eines dominant-thorakalen Atemmusters (reduzierte abdominelle Exkursionen) geachtet werden. Dieses könnte in der Einschätzung der Autoren zur Pathogenese der EILO beitragen. Ebenso beschreibt Depiazzi [34] ein gemeinsames Auftreten von EILO und auffälliger 
thorakaler Atmung und eine Besserung der EILO durch eine therapeutische Umstellung des Atemmusters. Bereits Petcu [39] beschrieb ein physiologisches Konzept zur Interaktion zwischen Zwerchfell und Larynx.

Geben die Patienten in der Anamnese paroxysmale Atemnot-Attacken außerhalb von körperlicher Belastung an, macht eine standardisierte Laufbandbelastung mit nachfolgenden repetitiven Lungenfunktionsuntersuchungen dann Sinn, wenn ein Asthma bronchiale differenzialdiagnostisch in Erwägung gezogen wird, obwohl die Ruhe-Lungenfunktion unauffällig und der Bronchospasmolysetest negativ waren. In dem Fall ist es sinnvoll, die vermuteten spezifischen Auslöser im klinischen Setting zu testen (z.B. durch Geruchsstimulation, induzierte Hyperventilation etc.).

Im Falle einer typischen Anamnese und einer Befund-Konstellation in der Laufband-Provokation mit frühem Beschwerdebeginn während der Belastung, raschem Abklingen nach Ende der Belastung und fehlenden Hinweisen in der Lungenfunktion auf eine EIB ist die Diagnose einer EILO hochwahrscheinlich. In diesem Setting werden im ERS-ELS-Positionspapier 2017 [2] eine Bestätigung der EILO-Diagnose und eine Zuordnung zur Ebene der Störung (supraglottisch versus glottisch) per Laryngoskopie unter körperlicher Belastung („,continuous laryngoscopy during exercise“, CLE-Test) empfohlen [40]. Da die CLE sehr aufwendig und das dafür erforderliche Setting nur in wenigen Zentren verfügbar ist, ist diese nur in besonders schweren bzw. unklaren Fällen erforderlich.

Bei Persistenz der Symptomatik sollten die Patienten an Zentren mit entsprechender Expertise überwiesen werden. Mittels 24h-pH-Metrie-/Impedanzmessung und Laryngoskopie können Hinweise auf einen laryngopharyngealen Reflux gewonnen werden, sodass sie insbesondere bei den nichtanstrengungsinduzierten ILO-Formen sinnvoll sein können, zumal hieraus eine therapeutische Interventionsmöglichkeit erwachsen kann. Problematisch ist jedoch, dass auch bei auffälliger $\mathrm{pH}$ Metrie/Impedanzmessung noch keineswegs eine Kausalität bewiesen ist. So wurde in Studien mit Asthmapatienten in bis zu $70 \%$ ein Reflux diagnostiziert. Es konnte aber bisher nicht konsistent gezeigt werden, dass eine Refluxbehandlung bei Asthmatikern mit oder ohne nachgewiesenen Reflux zu einer besseren Symptomkontrolle führt. Dieses kann für die ILO ebenfalls aus Mangel an Daten nicht beantwortet werden. In einigen VCD-Fallserien war eine PPI-Intervention effektiv [9, 29, 30]; möglicherweise unterscheiden sich diesbezüglich VCDVarianten und EILO signifikant. Bei allen ILO-Formen sollte außerdem eine Evaluation der psychosozialen Belastung erfolgen.

\section{Therapeutische Strategien}

Die Empfehlungen zum therapeutischen Vorgehen beruhen im Wesentlichen auf empirischen Erfahrungen, da randomisierte kontrollierte Studien speziell zu ILO bisher fehlen.

Ein wesentlicher therapeutischer Effekt zeigt sich bereits durch die Stellung der Diagnose, eine ausführliche Aufklärung über die Ursachen und das Hervorheben, dass selbst starke Beschwerden nie vital bedrohlich sind („reassurance“) [41]. Es ist extrem wichtig, den Betroffenen zu vermitteln, dass man ihre Beschwerden ernst nimmt, dass sie tatsächlich bestehen und nicht simuliert werden. Außerdem sollte betont werden, dass man sie nicht für psychiatrisch krank hält, sondern es sich um eine häufige „Fehlregulation“ handelt, die in aller Regel auch gut und ohne Medikamente behandelbar ist.

Die Visualisierung der Kehlkopf-Dysfunktion z. B. mittels Wach-Laryngoskopie oder Demonstrationsvideos hilft dem Patienten bei der Akzeptanz und dem Verständnis für das Krankheitsbild und nimmt die vorhandene Angst. Wach-Laryngoskopien können darüber hinaus im Zuge von Atemmuster-modifizierenden Therapieinterventionen als Biofeedback genutzt werden.

Atemphysiotherapeutische Strategien zielen auf die Vermeidung bzw. Kontrolle der ILO-Symptome ab [42, 43]. Mit dem „retraining“ eines physiologischen Atemmusters sollen die Neigung zu ILOEpisoden verringert und die Angstbehaf- tung der Symptome reduziert werden. Häufig werden bei ILO thorakal betonte Atemexkursionen mit bevorzugter Inspirationsstellung gesehen. Über Aufklärung und PEP-Atmung („Positive-expiratory-pressure"-Atmung) kann diese normalisiert werden. Das "retraining“ fokussiert auf eine Wiederherstellung einer physiologischen Nasenatmung und einer Zwerchfellatmung, inklusive einer Detonisierung von hypertonen myofaszialen Strukturen von Bauchdecke und Atempumpe, zusätzlich auf adäquate Atemzugvolumina, Atemfrequenz und Integration von endexspiratorischen Pausen. Ergänzend werden die Patienten in ihrer Wahrnehmung von abweichenden Atemmustern und Vorboten von ILO-Attacken geschult, um diese möglichst frühzeitig unterbrechen zu lernen. Selbsthilfetechniken bestehen in Kehlkopf-absenkenden und Kehlkopfentspannenden Übungen [44] mit „gähnender Einatmung mit geschlossenen Lippen " und Nasenatmung sowie bewusster Zwerchfellatmung. Kürzlich konnte in einer kleinen Studie an 28 Patienten gezeigt werden, dass ein inspiratorisches Atemmuskeltraining zu einer Reduktion der Beschwerden bei Patienten mit ILO führt [35]. Hecheln und "sniff“ (schnelles Einatmen nur durch die Nase) sollen reflexartig die Stimmlippen abduzieren und mögen sich dadurch ggf. zur Akuttherapie von VCD-Attacken eignen. Daneben werden Techniken zur Vermeidung von ILO-triggerndem Husten und Räuspern vermittelt. Bei einer sehr selektierten Patientengruppe mit per Wachlaryngoskopie gesicherter supraglottischer EILO, unbefriedigender Therapieansprache auf nichtoperative Therapien und hohem Leidensdruck (z. B. bei Leistungssportlern) hat sich eine die Kehlkopfstrukturen stabilisierende Laser-Intervention (sog. Supraglottoplastik) als effektiv und sicher erwiesen [45]. Vor solchen Interventionen sollten die konservativen, insbesondere atemphysiotherapeutischen Therapieversuche aus in EILO-Therapie erfahrener Hand ausgereizt und die Patienten immer an ein ausgewiesenes Zentrum zur Mitbeurteilung überwiesen werden.

Bei Vorliegen einer chronischen Rhinosinusitis als aggravierendem Faktor 
können gezielte antibiotische Behandlungen, sekretmobilisierende Maßnahmen wie regelmäßige Nasenspülungen sowie ggf. eine topische antiinflammatorische Therapie, z. B. mit Mometasonoder Fluticason-haltigen Nasensprays, hilfreich sein. Im Falle einer Koinzidenz von Asthma bronchiale und ILO sollte der Patient lernen, zwischen Asthmaund ILO-Symptomen zu differenzieren, um dann die unterschiedlichen Selbsthilfetechniken und Therapien gezielt einzusetzen. Die auf die ILO-Symptomatik zielenden Therapien sollten durch in der Behandlung von funktionellen Atemstörungen erfahrene Physiotherapeuten, Logopäden oder Stimm-/Sprach- und Sprechtherapeuten erfolgen. Die Rolle des Erlernens von Entspannungstechniken ist unklar; ein positiver Effekt ist sicher denkbar und insbesondere bei den nicht isoliert durch körperliche Anstrengung getriggerten ILO-Arten großzügig einzubeziehen. Demgegenüber steht aus Sicht der Autoren aber das Modifizieren des Atemmusters bei den EILOVarianten an erster Stelle.

Ist die Differenzierung zwischen „echtem" Asthma bronchiale und ILO-Symptomen ambulant nicht umsetzbar, sollte an eine Rehabilitationsmaßnahme mit genau dieser Zielsetzung in einer hierin erfahrenen Einrichtung gedacht werden.

Bei Hinweisen auf einen GÖR bzw. LPR oder einer sich als schwer therapierbar erweisenden VCD sollte eine medikamentöse bzw. diätetische Therapie versucht werden.

Der primär behandelnde Arzt sollte versuchen, mögliche psychosoziale Belastungssituationen aufzudecken und anzusprechen. Dies kann sehr häufig die Symptome positiv beeinflussen. In einzelnen Fällen ist eine psychotherapeutische oder psychiatrische Intervention geboten. Es besteht der Eindruck, dass sich die Arten der im Hintergrund bestehenden Psychopathologie zwischen den Entitäten EILO und (Ruhe-)VCD durchaus unterscheiden. Belastbare Daten existieren hierzu allerdings nicht.

Die Prognose der ILO ist als günstig zu bezeichnen, sobald die Diagnose gestellt und der Patient über das Wesen der ILO (nicht lebensgefährlich, bekanntes Störungsbild) aufgeklärt wurde [41].
Ansonsten drohen bei chronifizierten Krankheitsverläufen eine eingeschränkte soziale Teilhabe und Alltagsaktivität mit signifikant verminderter Lebensqualität. Die Folgen reichen von Sportabstinenz über Aufgabe von Karrieren im Leistungssport bis zu sozialem Rückzug durch Angst vor vital gefährdend eingestuften Atemnot-Episoden. Des Weiteren drohen Folgeprobleme durch inadäquate, hoch dosierte medikamentöse Asthma-Therapie. Dieses verdeutlicht die Wichtigkeit einer frühen Diagnosestellung und adäquaten Behandlung von Patienten mit EILO.

\section{Fazit für die Praxis}

- Wiederholt episodenhaft auftretende Atemnot ist im Kindes- und Jugendalter ein häufiges Symptom.

- Oft besteht eine funktionelle Ursache, dennoch wird die Diagnose häufig erst mit langer Latenz gestellt. - Körperliche Anstrengung ist ein typischer Auslöser. Es gilt in dem Fall, die zwei Hauptdifferenzialdiagnosen anstrengungsinduziertes Asthma bronchiale und EILO (Exercise-induced laryngeal obstruction) zu unterscheiden.

- Daneben existieren funktionelle Luftnot-Beschwerden ohne Anstrengungsbezug (z. B. bei VCD); Unterschiede in Pathophysiologie und Therapie im Vergleich zur EILO sind zu vermuten.

- Es stehen wirksame Therapie-Strategien mit dem Grundgedanken, das Atemmuster zu modifizieren, zur Verfügung.

- Die Rolle aggravierender Faktoren wie gastroösophagealer (laryngopharyngealer) Reflux oder Laryngomalazie ist erst rudimentär verstanden.

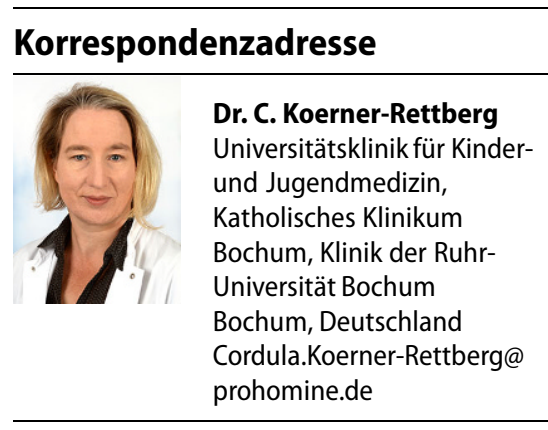

Funding. Open Access funding enabled and organized by Projekt DEAL.

\section{Einhaltung ethischer Richtlinien}

Interessenkonflikt. S. Dillenhöfer, B. Hinrichs, A. Kohl, M. Kuhnigk, R. Maas, D. Pfeiffer-Kascha, T. Rutt, A. Schlegtendal, J. Seidenberg, T. Spindler, C. Suerbaum, B. Wilmsmeyer, S. Zeidler und C. Koerner-Rettberg geben an, dass kein Interessenkonflikt besteht.

Für diesen Beitrag wurden von den Autoren keine Studien an Menschen oder Tieren durchgeführt. Für die aufgeführten Studien gelten die jeweils dort angegebenen ethischen Richtlinien.

Open Access. Dieser Artikel wird unter der Creative Commons Namensnennung 4.0 International Lizenz veröffentlicht, welche die Nutzung, Vervielfältigung Bearbeitung, Verbreitung und Wiedergabe in jeglichem Medium und Format erlaubt, sofern Sie den/die ursprünglichen Autor(en) und die Quelle ordnungsgemäß nennen, einen Link zur Creative Commons Lizenz beifügen und angeben, ob Änderungen vorgenommen wurden.

Die in diesem Artikel enthaltenen Bilder und sonstiges Drittmaterial unterliegen ebenfalls der genannten Creative Commons Lizenz, sofern sich aus der Abbildungslegende nichts anderes ergibt. Sofern das betreffende Material nicht unter der genannten Creative Commons Lizenz steht und die betreffende Handlung nicht nach gesetzlichen Vorschriften erlaubt ist, ist für die oben aufgeführten Weiterverwendungen des Materials die Einwilligung des jeweiligen Rechteinhabers einzuholen.

Weitere Details zur Lizenz entnehmen Sie bitte der Lizenzinformation auf http://creativecommons.org/ licenses/by/4.0/deed.de.

\section{Literatur}

1. Niggemann B, Grüber C (2014) Dysfunktionelle respiratorische Symptome bei Kindern u. Jugendlichen. Consilium Pneumol 1 (Sonderausgabe 2014):1-20

2. Halvorsen et al (2017) Inducible laryngeal obstruction: an official jointERS and ELS statement. EurRespir J50:1602221

3. Newman KB, Dubester SN (1994) Vocal cord dysfunction: masquerader of asthma. Seminars Respir Crit Care Med 15:161-167

4. Christensen PM, Heimdal JH (2015) ERS/ELS/ACCP 2013 International consensus conference nomen- 
clature on inducible laryngeal obstructions. Eur Respir Rev 24:445-4502

5. Johansson H, Norlander K, Berglund L, Janson C, Malinovschi A, Nordvall L, Nordang L, Emtner M (2015) Prevalence of exercise-induced bronchoconstriction and exercise-induced laryngeal obstruction in a general adolescent population. Thorax 70:57-63

6. Morris MJ, Grbach VX, Deal LE, Boyd SYN, Morgan JA, Johnson JE (2002) Evaluation of exertional dyspnoe in the active duty patient: the diagnostic approach and the utility of clinical testing. Mil Med 167(4):281-288

7. Seear M, Wensley D, West N (2005) How accurate is the diagnosis of exercise induced asthma among Vancouver schoolchildren? Arch Dis Child 90:898-902

8. Abu-Hasan M, Tannous B, Weinberger M (2005) Exercise-induced dyspnoe in children and adolescents: if not asthma then what? Ann Allergy Asthma Immunol 94(3):366-371

9. George S, Suresh S (2019) Vocal cord dysfunction: Analysis of 27 cases and updated review on pathophysiology and management. Int Arch Otorhinolaryngol 23:125-130

10. Brugman S (2003) The many faces of vocal cord dysfunction. What 36 years of literature tells us. Am JRespir Crit Care Med 167:A588

11. Morris MJ, Allan PF, Perkins PJ (2006) Vocal cord dysfunction: aetiologies and treatment. Clin Pulm Med 13:73-86

12. Rundell KW, Spiering BA (2003) Inspiratory stridor in elite athletes. Chest 123:468-474

13. Nielsen EW, Hull JH, Backer V (2013) High prevalence of exercise-induced laryngeal obstruction in athletes. Med Sci Sports Exerc 45(11):2030-2035

14. Christopher KL, Wood RP, Eckert RC, Blager FB, Raney RA, Souhrada JF (1983) Vocal cord dysfunction presenting as asthma. N Engl J Med 308:1566-1570

15. Gavin LA, Wamboldt M, Brugman S, Roesler TA, Wamboldt F (1998) Psychological and family characteristics of adolescents with vocal cord dysfunction. J Asthma 35(5):409-417

16. Leo RJ, Konakanchi R (1999) Psychogenic respiratory distress: A case of paradoxical vocal cord dysfunction and literature review. Prim Care Companion JClin Psychiatry 1(02):39-46

17. Lacy TJ, McManis SE (1994) Psychogenic stridor. Gen Hosp Psychiatry 16(03):213-223

18. Powell DM, Karanfilov BI, Beechler KB, Treole K, Trudeau MD, Forrest LA (2000) Paradoxical vocal cord dysfunction in juveniles. Arch Otolaryngol Head Neck Surg 126(01):29-34

19. Bucca C, Rolla G, Scappaticci E, Chiampo F, Bugiani M, Magnano M, D'Alberto M (1995) Extrathoracic and intrathoracic airway responsiveness in sinusitis. J Allergy Clin Immunol 95(1 Pt1):52-59

20. Merati AL, Lim HJ, UlualpSO, ToohillRJ (2005) Metaanalysis of upper probe measurements in normal subjects and patients with laryngopharyngeal reflux. Ann Otol Rhinol Laryngol 114(3):177-182

21. Schnatz PF, Castell JA (1996) Pulmonary symptoms associated with gastroesophageal reflux: use of ambulatory $\mathrm{pH}$ monitoring to diagnose and to direct therapy. Am J Gastroenterol 91(9):1715-1718

22. Harding SM (2003) Recent clinical investigations examining the association of asthma and gastroesophageal reflux. Am J Med 115(3A):39S-44S

23. Kenn K, Balkissoon R(2011) Vocal cord dysfunction: what do we know? Eur Respir J37:194-200
24. Schlegtendal A, Koerner-Rettberg C (2019) Dysfunktionelle respiratorische Symptome. Pneumologe 16:33-39

25. Perkner JJ, Fennelly KP, Balkissoon R, Bartelson BB, Ruttenber AJ, Wood RP, Newman LS (1998) Irritantassociated vocal cord dysfunction. JOccup Environ Med 40:136-143

26. Perkins PJ, Morris MJ (2002) Vocal cord dysfunction induced by methacholine challenge testing. Chest 122(6):1988-1993

27. Powell J, Cocks HC (2013) Mucosal changes in laryngopharyngeal reflux — prevalence, sensitivity, specificity and assessment. Laryngoscope 123(4):985-991

28. Johnston N, Bulmer D, Gill GA, Panetti M, Ross PE, Pearson JP, Pignatelli M, Axford SE, Dettmar PW, Koufman JA (2003) Cell biology of laryngeal epithelial defenses in health and disease: further studies. Ann Otol Rhinol Laryngol 112(6):481-491

29. Patigaroo SA, Hashmi SF, Hasan SA (2011) Clinical manifestations and role of proton pump inhibitors in the management of laryngopharyngeal reflux. Indian J Otolaryngol Head Neck Surg 63(29):182-189

30. Boris M, Goldblatt A, Krigsman A (2002) Laryngeal dysfunction: a common cause of respiratory distress. Often misdiagnosed as asthma and responsive to antireflux therapy. Allergy Asthma Proc 23:133-139

31. Newman KB, Mason UG 3rd, Schmaling KB (1995) Clinical features of vocal cord dysfunction. Am J Respir Crit Care Med 152(4 Pt 1):1382-1386

32. Yelken K, Yilmaz A, Guven M, Eyibilen A, Aladag I (2009) Paradoxical vocal fold motion dysfunction in asthma patients. Respirology 14(5):729-733

33. Hilland M, Roksund OD, Sandvik L, Haaland O, Aarstad HJ, Halvorsen T, Heimdal JH (2016) Congenital laryngomalacia is related to exerciseinduced laryngeal obstruction in adolescence. Arch Dis Child 101:443-448

34. Depiazzi J, Everard ML (2016) Dysfunctional breathing and reaching one's physiological limit as causes of exercise-induced dyspnoe. Breathe 12:120-129

35. Sandnes A, Andersen T, Clemm HH, Hilland M, Vollsaeter M, Heimdal JH, Eide GE, Halvorsen T, Roksund OD (2019) Exercise-induced laryngeal obstruction in athletes treated with inspiratory muscle training. BMJ Open Sport Exerc Med 5(1):e436. https://doi.org/10.1136/bmjsem2018-000436

36. Kenn K, Schmitz M (1997) Prevalence of vocal cord dysfunction in patients with dyspnoe. First prospective clinical study (abstract). Am J Respir Crit Care Med 155:A965

37. Fowler SJ, Thurston A, Chesworth B, Cheng V, Constantinou P, Vyas A, Lillie S, Haines J (2015) The VCDQ a questionnaire for symptom monitoring in vocal cord dysfunction. Clin Exp Allergy 45:1406-1411

38. Pinto LHE, Aun MV, Cukier-Blaj $S$, Stelmach $R$, Cukier A, Kalil J, Agondi RC, Giavina-Bianchi P (2016) Vocal cord dysfunction diagnosis may be improved by a screening check list. Allergol Int 65:180-185

39. Petcu LG, Sasaki CT (1991) Laryngeal anatomy and physiology. Clin Chest Med 12:415-423

40. Heimdal JH, Roksund OD, Halvorsen T (2006) Continuous laryngoscopy exercise test: a method for visualizing laryngeal dysfunction during exercise. Laryngoscope 116:52-57

41. Røksund OD, Heimdal JH, Clemm H, Vollsaeter $M$ Halvorsen T (2017) Exercise induces laryngeal obstruction: diagnostics and treatment. Paediatr Respir Rev 21:86-94

42. Barker NJ, Elphick H, Everard ML (2016) The impact of a dedicated physiotherapist clinic for children with dysfunctional breathing. ERJ Open Res 2(3):00103-2015

43. Weise S, Kardos P, Pfeiffer-Kascha D, Worth H(2019) Empfehlungen zur Atemphysiotherapie, 3. Aufl. Deutsche Atemwegsliga u. Dustri-Verlag Dr. Karl Feistle, , S14. ISBN 978-3-87185-545-0

44. Patel RR et al (2015) Evidence-based systematic review: effects of speech-language pathology treatment for individuals with paradoxical vocal fold motion. Am J Speech Lang Pathol 24(3):566-584. https://doi.org/10.1044/2015_ AJSLP-14-0120

45. Sandnes A, Hilland M, Vollsaeter M, Andersen T, Engesaeter IO, Sandvik L, Heimdal JH, Halvorsen T, Eide GE, Roksund OD, Clemm HH (2019) Severe exercise-induced laryngeal obstruction treated with supraglottoplasty. Front Surg 6:44. https:// doi.org/10.3389/fsurg.2019.00044 